\title{
EDITORIAL
}

\section{Prognosis of ventilator-associated pneumonia: what lies beneath}

\author{
S. Krüger*, D. Frechen* and S. Ewig ${ }^{\#}$
}

$\mathbf{V}$ entilator-associated pneumonia (VAP) continues to be a major problem in the care of intensive care unit (ICU) patients. The incidence of VAP in mechanically ventilated patients is high, ranging from 10 to $30 \%$. The clinical symptoms of VAP are ambiguous and there is no generally accepted gold standard for the diagnosis of VAP. The associated mortality is still high at $30-40 \%$ [1, 2]. Established clinical scores and biomarkers are only of limited value in terms of diagnosis and prognosis. Since VAP is an infectious disease we traditionally use biomarkers of infection for diagnostic and prognostic purposes. Inflammatory markers such as leukocyte count and C-reactive protein (CRP) are still widely used in the diagnosis of VAP, although it is well known that sensitivity in the ICU setting is highly limited.

Until recently, only procalcitonin as a more accurate marker of bacterial infection turned out to be of clinical value. Procalcitonin is useful for the guidance of antibiotic therapy in community-acquired pneumonia (CAP), which has been shown by several studies of the Basel group in recent years $[3,4]$. NOBRE et al. [5] were the first to demonstrate a median reduction of antibiotic treatment of 4 days (six versus 10) following a procalcitonin-guided strategy as opposed to a clinical strategy in patients with VAP. The ProVAP trial demonstrated that procalcitonin guidance can reduce the duration of antibiotic therapy in patients with VAP. In the ProVAP trial STOLZ et al. [6] performed a multicentre, randomised, controlled trial with 101 patients with VAP. Patients were either assigned to an antibiotic discontinuation strategy according to American Thoracic Society/Infectious Diseases Society of America guidelines (control group) or to a strategy using serial serum procalcitonin measurements (procalcitonin group). The number of antibiotic-free days patients were alive 28 days after VAP onset was significantly shorter in the procalcitonin-guided group compared with the reference group (9.5 days versus 13 days), translating into a reduction in the overall duration of antibiotic therapy of $27 \%$ in the procalcitonin-guided group.

In the current issue of the European Respiratory Journal, BOECK et al. [7] bring new insights for the understanding of prognostic

\footnotetext{
*Medical Clinic I, University Clinic RWTH Aachen, Aachen. ${ }^{\#}$ Thoraxzentrum Ruhrgebiet, Kliniken für Pneumologie und Infektiologie, Ev. Krankenhaus Herne und Augusta Kranken-Anstalt, Bochum, Germany.

CORRESPONDENCE: S. Krüger, Medical Clinic I, University Hospital, RWTH University Aachen, Pauwelsstr. 30, D-52057 Aachen, Germany. E-mail: stkrueger@ukaachen.de
}

factors in VAP. The authors performed a subanalysis of the ProVAP trial investigating the prognostic value of the cardiovascular marker midregional (MR) pro-atrial natriuretic peptide (MR-proANP) and the inflammatory marker procalcitonin. They compared the prognostic value of the biomarkers with that of the clinical severity scores sequential organ failure assessment (SOFA) and simplified acute physiology score (SAPS II) for the prediction of death at 28 days. They found that both MR-proANP and procalcitonin on VAP onset were elevated in nonsurvivors compared with survivors. However, their slope of decline was different. MR-proANP was the best predictor for survival. The addition of MR-proANP and procalcitonin to the clinical scores SAPS II and SOFA significantly improved the predictive value of the scores.

Are biomarkers useful for the prediction of survival in patients with VAP? To date, reports in the literature provided conflicting results. One observational study by HiLlas et al. [8] found that there was no difference in survivors and nonsurvivors in CRP values, whereas procalcitonin levels on day 1 and 7 were higher in nonsurvivors. However, neither CRP nor procalcitonin threshold values nor their kinetics were good predictors of survival. The only factor predicting the development of septic shock was the SOFA score on day 1. In contrast, Po'voA et al. [9] found serial CRP measurements a useful tool to identify poor outcome from day 4 onwards. Likewise, LUYT et al. [10] identified a strong predictive potential for procalcitonin for the prediction of a composite end-point survival, recurrence and extrapulmonary infection. Finally, SELIGMan et al. [11] found CRP and procalcitonin kinetics (decreasing values during follow-up) predictive of survival.

The main question when we look at the mortality of a disease is: what are the reasons why patients die? So, why do patients with VAP die? Besides traumatic brain injury/subarachnoid haemorrhage, the causes of death in this study were respiratory failure/acute respiratory distress syndrome, septic shock, cardiogenic shock, multiorgan failure or acute liver failure. Each of these conditions reflects a highly complex inflammatory and cardiovascular response, and it is tempting to focus the search for predictors on biomarkers which best reflect inflammation and cardiovascular compromise. And what biomarkers are the best indicators of cardiovascular problems and circulatory failure at the moment? Natriuretic peptides such as brain natriuretic peptide or atrial natriuretic peptide (ANP)!

Sepsis and cardiovascular complications are the main causes of death in VAP. There are some mechanisms that might be 
responsible for the marked increase in MR-proANP in patients with VAP. Plasma concentrations of ANP are increased in patients with chronic heart failure, renal disease and sepsis, and predict impaired left ventricular ejection fraction [12]. This is supported by the results of the present study that showed, although only moderate, significant positive correlations of MR-proANP with cardiac, pulmonary and renal comorbidities. In patients with VAP, cardiac failure and consecutive elevation of MR-proANP might be due to underlying pre-existing cardiac disease or septic cardiomyopathy. In studies on biomarkers by the German CAP Competence Network (CAPNETZ) trial, they have demonstrated that the cardiovascular biomarkers proANP, pro-arginin vasopressin (proAVP), pro-endothelin-1 and pro-adrenomedullin (proADM) are good predictors of short- and long-term outcome in CAP and even superior to inflammatory markers [13-15]. Interestingly, we found that proAVP, MR-proANP and MR-proADM are predictive for mortality in patients with CAP independently of a pre-existing diagnosis of chronic heart disease or heart failure. In the setting of pneumonia, irrespective of if it is CAP or VAP, underlying and possibly previously unknown cardiovascular or renal disease may be aggravated due to acute inflammatory activation. Although the underlying cardiovascular disease might not appear directly clinically evident, it could result in higher serum levels of MR-proANP. This hypothesis is supported by our finding that MR-proANP was a stronger predictor of mortality compared with the history of congestive heart failure or other comorbidities alone. The study by BOECK et al. [7] demonstrated the same phenomenon in VAP which we found in CAP. In their study, MR-proANP was the strongest predictor of mortality, whereas the frequency of cardiac, pulmonary and renal comorbidities was not different between survivors and nonsurvivors. Taken together, the elevation of MR-proANP might be attributable to hidden cardiovascular comorbidities or to cardiovascular dysfunction as a consequence of the acute infection of VAP. Thus, the clinical benefit of MR-proANP as a cardiovascular marker could be the identification of VAP patients with an underlying cardiovascular morbidity or severe sepsis, both imposing the patient to increased risk of death.

What could biomarkers in VAP be used for in the future? Procalcitonin could be used for a better diagnosis of VAP and sepsis and for the guidance of antibiotic therapy, a very important issue in order to reduce selection pressure and multiresistance. MR-proANP could be used for the identification of patients with a high cardiovascular risk. In these patients, increased attention to possible cardiovascular disease and closer medical follow-up may be indicated and could result in a better outcome. Whether the better predictive potential of MRproANP and procalcitonin translates into clinically relevant prediction tools of mortality from VAP remains to be determined. In fact, in the present study as well as in recent CAP studies, it could be shown that clinical scores and biomarkers can both be merged and result in superior predictions of mortality [7, 13-15]. A combination of a clinical score such as SOFA or SAPS II with MR-proANP for risk assessment and procalcitonin for guidance of antibiotic therapy is an attractive approach awaiting validation in independent future cohorts.

Scores have one problem as one can see again in this study: they are composed of several parameters, which make the calculation of the scores time consuming. The SOFA and SAPS II score are validated for the ICU. Although they include six single parameters in the SOFA and 15 single parameters in the SAPS II score with the intention to compose a complex system of different pathophysiological aspects of a disease treated in the ICU, the scores are inferior to MR-proANP as a single biomarker to predict mortality. There is only one sensible explanation why a biomarker like MR-proANP might be better compared to a complex score: the biomarker is not an indicator for one exclusive disease or single physiological disturbance. A relevant biomarker for prognosis, such as MR-proANP, reflects not a single but multiple pathophysiological aspect of a disease. There are other biomarkers on the horizon with multiplex functions. The most promising one seems to be MRproADM which possesses not only cardiovascular activity but also anti-inflammatory and anti-bacterial functions, which might be very helpful especially in the difficult setting of an ICU. Thus, we have to await the results of future studies in well defined cohorts applying different sets of biomarkers and combinations of scores and biomarkers to clarify the role this new approach may have in clinical practice.

\section{STATEMENT OF INTEREST}

A statement of interest for S. Ewig can be found at www.erj. ersjournals.com/site/misc/statements.xhtml

\section{REFERENCES}

1 Torres A, Ewig S, Lode H, et al. Defining, treating and preventing hospital acquired pneumonia: European perspective. Intensive Care Med 2009; 35: 9-29.

2 Torres A, Ewig S, Lode H, et al. Hospital-acquired pneumonia in Europe. Eur Respir J 2009; 33: 951-952.

3 Christ-Crain M, Jaccard-Stolz D, Bingisser R, et al. Effect of procalcitonin-guided treatment on antibiotic use and outcome in lower respiratory tract infections: cluster-randomised, singleblinded intervention trial. Lancet 2004; 363: 600-607.

4 Christ-Crain M, Stolz D, Bingisser R, et al. Procalcitonin guidance of antibiotic therapy in community-acquired pneumonia: a randomized trial. Am J Respir Crit Care Med 2006; 174: 84-93.

5 Nobre V, Harbarth S, Graf JD, et al. Use of procalcitonin to shorten antibiotic treatment duration in septic patients: a randomized trial. Am J Respir Crit Care Med 2008; 177: 498-505.

6 Stolz D, Smyrnios N, Eggimann P, et al. Procalcitonin for reduced antibiotic exposure in ventilator-associated pneumonia: a randomised study. Eur Respir J 2009; 34: 1364-1375.

7 Boeck L, Eggimann P, Smyrnios N, et al. Midregional pro-atrial natriuretic peptide and procalcitonin improve survival prediction in VAP. Eur Respir J 2011; 37: 595-603.

8 Hillas G, Vassilakopoulos T, Plantza P, et al. C-reactive protein and procalcitonin as predictors of survival and septic shock in ventilator-associated pneumonia. Eur Respir J 2010; 35: 805-811.

9 Po'voa P, Coelho L, Almeida E, et al. Early identification of intensive care unit-acquired infections with daily monitoring of $C$ reactive protein: a prospective observational study. Crit Care 2006; 10: R63.

10 Luyt CE, Gue'rin V, Combes A, et al. Procalcitonin kinetics as a prognostic marker of ventilator-associated pneumonia. Am J Respir Crit Care Med 2005; 171: 48-53.

11 Seligman R, Meisne M, Lisboa TC, et al. Decreases in procalcitonin and C-reactive protein are strong predictors of survival in ventilator-associated pneumonia. Crit Care 2006; 10: R125.

12 Morgenthaler NG, Struck J, Christ-Crain M, et al. Pro-atrial natriuretic peptide is a prognostic marker in sepsis, similar to 
the APACHE II score: an observational study. Crit Care 2003; 9: R37-R45.

13 Krüger S, Ewig S, Kunde J, et al. Pro-atrial natriuretic peptide and pro-vasopressin to predict short- and long-term survival in community-acquired pneumonia. Results from the German competence network CAPNETZ. Thorax 2010; 65: 208-214
14 Krüger S, Ewig S, Giersdorf S, et al. Cardiovascular and inflammatory biomarkers to predict short- and long-term survival in community-acquired pneumonia. Am J Respir Crit Care Med 2010; 182: 1426-1434.

15 Krüger S, Ewig S, Marre R, et al. Procalcitonin predicts patients at low risk of death from community-acquired pneumonia across all CRB-65 classes. Eur Respir J 2008; 31: 349-355. 